\title{
RUPTURE LINE IN A TWO-LAYER NON-COHESIVE MEDIUM
}

\author{
J. DRNOVŠEK*
}

\begin{abstract}
The author presents the analytical and the graphical method for determination of the direction and of the stress in the rupture line on one side of the contact between two different non-cohesive media, provided that the following values are known: the shear angles of both media that are assumed to obey Coulomb's failure law, the dilatation angles of both media as well as the stress state and the direction of the failure line on the other side of the contact. The analytical solution has been deduced from the limiting equilibrium conditions for the prismatic contact element whose surfaces are formed by the strain characteristics of both media appearing in the narrow rupture zone in which-according to Bent Hansen-the line rupture develops. The graphical solution can be obtained by constructing the corresponding Mohr's stress circles. The analytical as well as the graphical solution can be used also in order to get the dilation angles provided that the directions of the rupture line at the contact and the magnitude of the normal stress in the contact were determined in appropriate model tests.
\end{abstract}

\section{INTRODUCTION}

Line ruptures are assumed to occur in narrow rupture zones separating rigid domains (Bent Hansen, 1958). As linear strains in the directions of limiting rupture lines are zero, these rupture lines coincide with strain characteristics.

In Mohr's strain circle the strain characteristics are represented, in Fig. 1, by the points $a$ and $b$, and in Mohr's circle of limiting stress state of a non-cohesive medium the stress characteristics are given, in Fig. 2, by the points $m$ and $n$.

While along stress characteristics the shear angle is fully mobilized, the inclination of stress vectors towards normals to strain characteristics is less than or equal to the shear angle: $\phi^{\prime} \leq \phi$. The relationship between the angles $\phi$ and $\phi^{\prime}$ is governed by the dilation angle $\nu$ (see Fig. 2) and given by the equation:

$$
\sin \phi=\frac{\sin \phi^{\prime}}{\cos \left(\phi^{\prime}-\nu\right)}
$$

When $\nu=\phi^{\prime}$, the angles $\phi$ and $\phi^{\prime}$ coincide:

$$
\phi \equiv \phi^{\prime}
$$

* University of Ljubljana, Yugoslavia.

Written discussions on this paper should be submitted before October 1, 1970. 


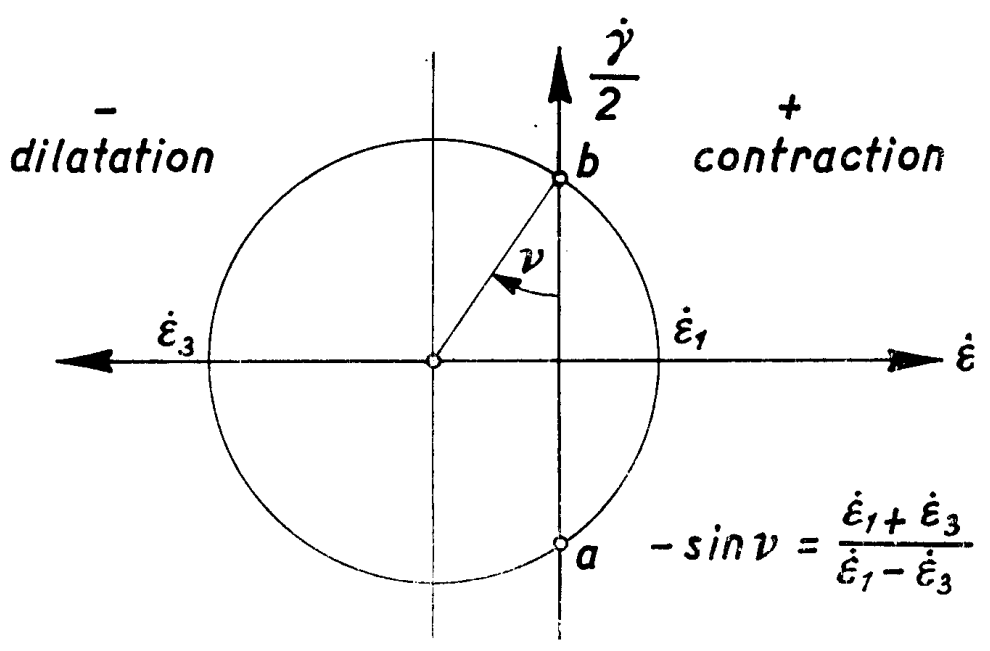

Fig. 1. Mohr's strain circle

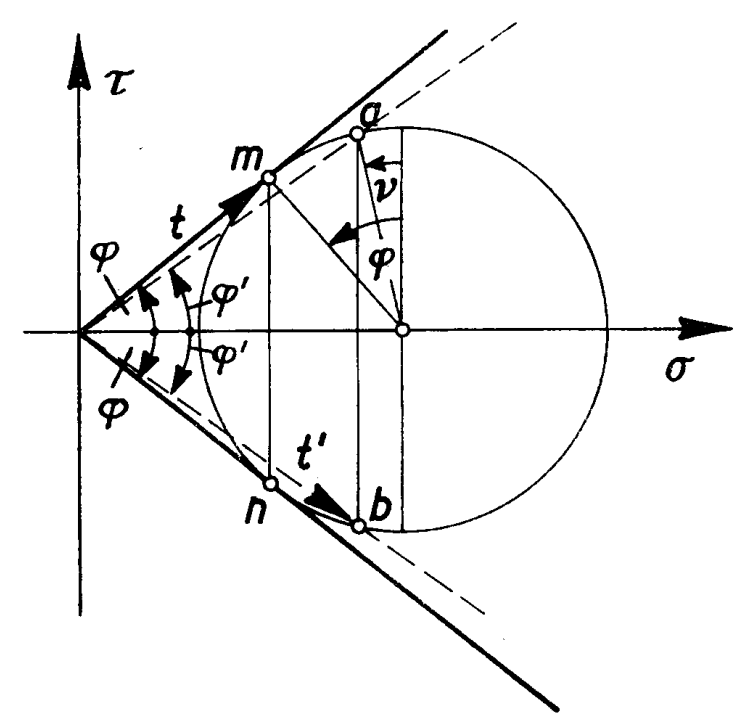

Fig. 2. Mohr's limiting stress circle

\section{ANALYTICAL SOLUTION}

In narrow rupture zones of line ruptures the strain characteristics form angles $\frac{\pi}{2} \pm \nu$ (see Fig. 1). Considering different shear angles $\phi_{1}$ and $\phi_{2}$ and different bulk densities $\gamma_{1}$ and $\gamma_{2}$ of two horizontal layers, the rupture lines must be assumed to be broken in the contact of the layers. Fig. 3 represents the corresponding part of the narrow rupture zone for the case of an upward displacement. In the case of an downward displacement of the zone the angles $\phi_{1}^{\prime}$ and ${\phi_{2}}^{\prime}$ get negative values.

Let us consider the equilibrium conditions for the soil body consisting of two triangular prisms having as common surface the contact surface $c \cdot d(d \rightarrow \infty)$ of the layers in the rupture zone and limited by strain characteristics $a_{1}, b_{1}$ and $a_{2}, b_{2}$ as shown in Fig. 3. The body is subjected to volume forces $G_{1}$ and $G_{2}$. 


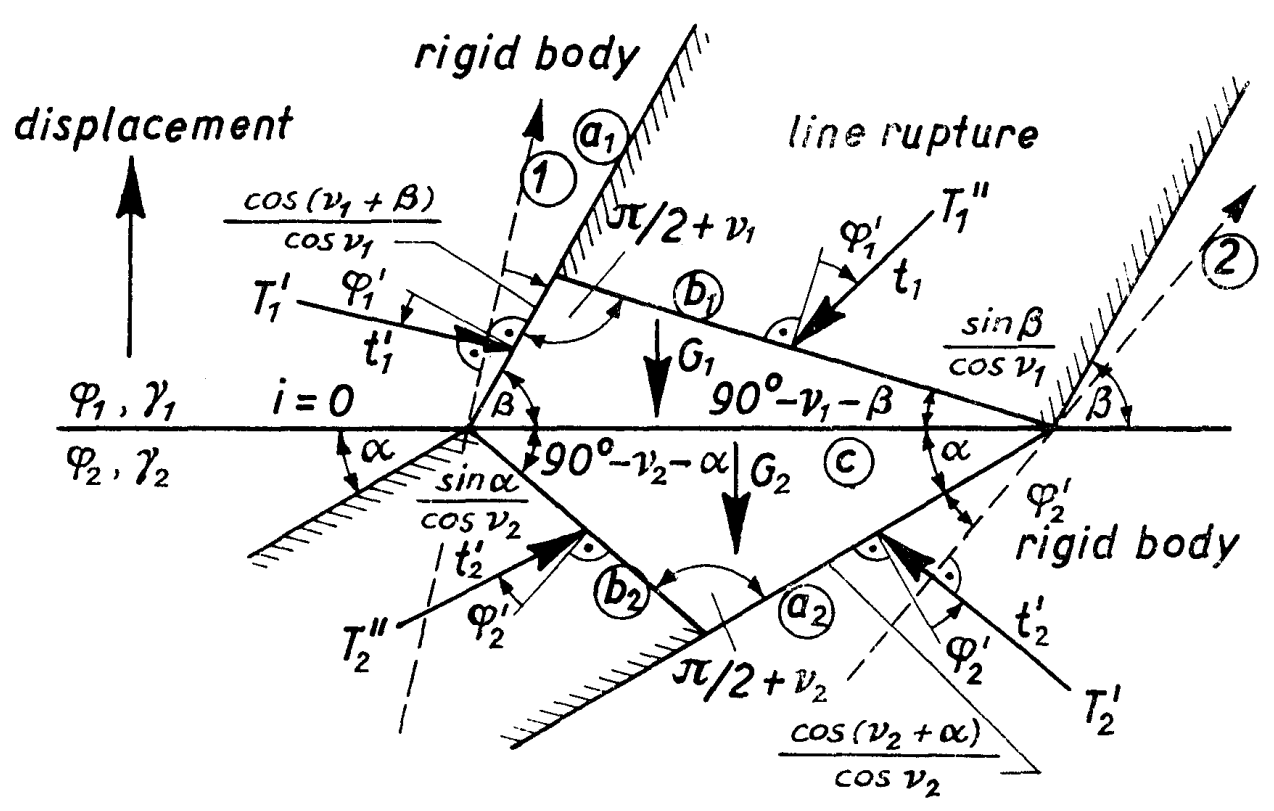

Fig. 3. Narrow rupture zone at the contact of two layers

$$
\begin{aligned}
& G_{1}=c^{2} d \frac{\gamma_{1}}{2} \frac{\sin \beta \cos \left(\nu_{1}+\beta\right)}{\cos \nu_{1}}, \\
& G_{2}=c^{2} d \frac{\gamma_{2}}{2} \frac{\sin \alpha \cos \left(\nu_{2}+\alpha\right)}{\cos \nu_{2}},
\end{aligned}
$$

and to surface forces

$$
\begin{aligned}
& T_{1}^{\prime}={t_{1}}^{\prime} \frac{\cos \left(\nu_{1}+\beta\right)}{\cos \nu_{1}} c d \\
& T_{1}^{\prime \prime}=t_{1}^{\prime \prime} \frac{\sin \beta}{\cos \nu_{1}} c d \\
& T_{2}^{\prime}=t_{2}{ }^{\prime} \frac{\cos \left(\nu_{2}+\alpha\right)}{\cos \nu_{2}} c d \\
& T_{2}^{\prime \prime}=t_{2}^{\prime \prime} \frac{\sin \alpha}{\cos \nu_{2}} c d
\end{aligned}
$$

As the width $(c)$ of the repture zone has been assumed to be very small, the volume forces can be neglected when forming the equilibrium conditions. In this way the width of the zone gets eliminated in the solution. The equilibrium conditions for moments then yield the equations

$$
\begin{aligned}
& t_{1}{ }^{\prime}=t_{1}{ }^{\prime \prime}=t_{1} \\
& t_{2}{ }^{\prime}=t_{2}{ }^{\prime \prime}=t_{2}
\end{aligned}
$$

Considering the above solution, the application of the equilibrium conditions for forces to the axes (1) and (2) (see Fig. 3) gives the following two equations: 


$$
\begin{aligned}
\frac{t_{1}}{t_{2}}= & \frac{1}{\cos \nu_{2} \sin \beta \cos \left(2 \phi_{1}{ }^{\prime}-\nu_{1}\right)}\left\{\operatorname { c o s } \nu _ { 1 } \left[\sin \alpha \cos \left(\beta-\alpha+\phi_{1}{ }^{\prime}+\phi_{2}{ }^{\prime}-\nu_{2}\right)\right.\right. \\
& \left.\left.+\cos \left(\alpha+\nu_{2}\right) \sin \left(\beta-\alpha+\phi_{1}{ }^{\prime}-\phi_{2}{ }^{\prime}\right)\right]\right\} \\
\frac{t_{1}}{t_{2}}= & \cos \nu_{1} \sin \alpha \cos \left(2{\phi_{2}}^{\prime}-\nu_{2}\right)\left\{1 / \cos \nu_{2}\left[\sin \beta \cos \left(\alpha-\beta+{\phi_{1}}^{\prime}+{\phi_{2}}^{\prime}-\nu_{1}\right)\right.\right. \\
& \left.\left.-\cos \left(\nu_{1}+\beta\right) \sin \left(\beta-\alpha+{\phi_{1}}^{\prime}-\phi_{2}\right)\right]\right\}
\end{aligned}
$$

If the strength parameters $\phi_{1}, \phi_{2}$ and $\nu_{1}, \nu_{2}$ have been obtained by appropriate testing procedure, and if the values $t_{1}$ and $\beta$ are assumed to be known, Equations (7) and (8) yield the values $t_{2}$ and $\alpha$.

\section{GRAPHICAL SOLUTION}

The graphical solution of the problem can be obtained by using Mohr's circles of stress. The solution is governed by the condition that in the contact surface of two layers the same stress vector $\overrightarrow{p_{d}}\left(\sigma_{d}, \tau_{d}\right)$ must be obtained if computing it from either the stress state of the upper or of the lower triangle shown in Fig. 3. For the case of the upward displacement of the narrow rupture zone the graphical construction is presented in Fig. 4.

The strength parameters $\phi_{1}, \phi_{2}$ and $\nu_{1}, \nu_{2}$ as well as the values $t_{1}$ and $\beta$ shall again be assumed to be known. Thus the circle touching, in point 1 , the failure envelope $\phi_{1}$ of the upper layer defines the stress state in the triangle above the contact provided that the magnitude of the stress vector $\overrightarrow{02}: \overline{02}=t_{1}$. The mirror image of the point 2 about the axis $\sigma$ is the point 3. The straight line passing through the point 3 intersects the stress circle in the pole $P_{1}$. The horizontal direction drawn through the pole $P_{1}$ intersects the Mohr circle at the point 4 , and its mirror image 5 defines the stress vector $\overrightarrow{05}$ acting in the contact surface of both layers.

The stress state in the lower layer is then given by the stress circle passing through the points 4 and 5 and touching the failure envelope $\phi_{2}$ of the lower layer in the point 6 . The angle $\phi_{2}-\nu_{2}=\Varangle\left(6-0_{1}-7\right)$ determines the stress vector $\overrightarrow{t_{2}}=\overrightarrow{07}$. The inclination $\alpha$ of the rupture line in the lower layer (see Fig. 3) can be obtained by connecting the mirror image of the point 7 , i.e., point 8 , with the pole $P_{2}$ of the stress circle whose centre is the point $0_{2}$. The pole $P_{2}$ lies on the horizontal drawn through the point 4 .

The author has presented a method of determining the direction of strain characteristics and the stress state on one side of the horizontal surface separating two layers of different strengths, provided that the strength characteristics of both layers and the stress state on the other side of the contact are known. According to Bent Hansen, the line rupture was assumed to occur in a narrow rupture zone. The strain characteristics have been allowed to deviate from stress characteristics. The dilatation angle can be obtained by measuring, in plane strain conditions, the strains preceding failure in both principal directions.

If the directions of strain characteristics limiting narrow rupture zones of line ruptures were obtained by model tests made with two media of known shear angles, the procedure 


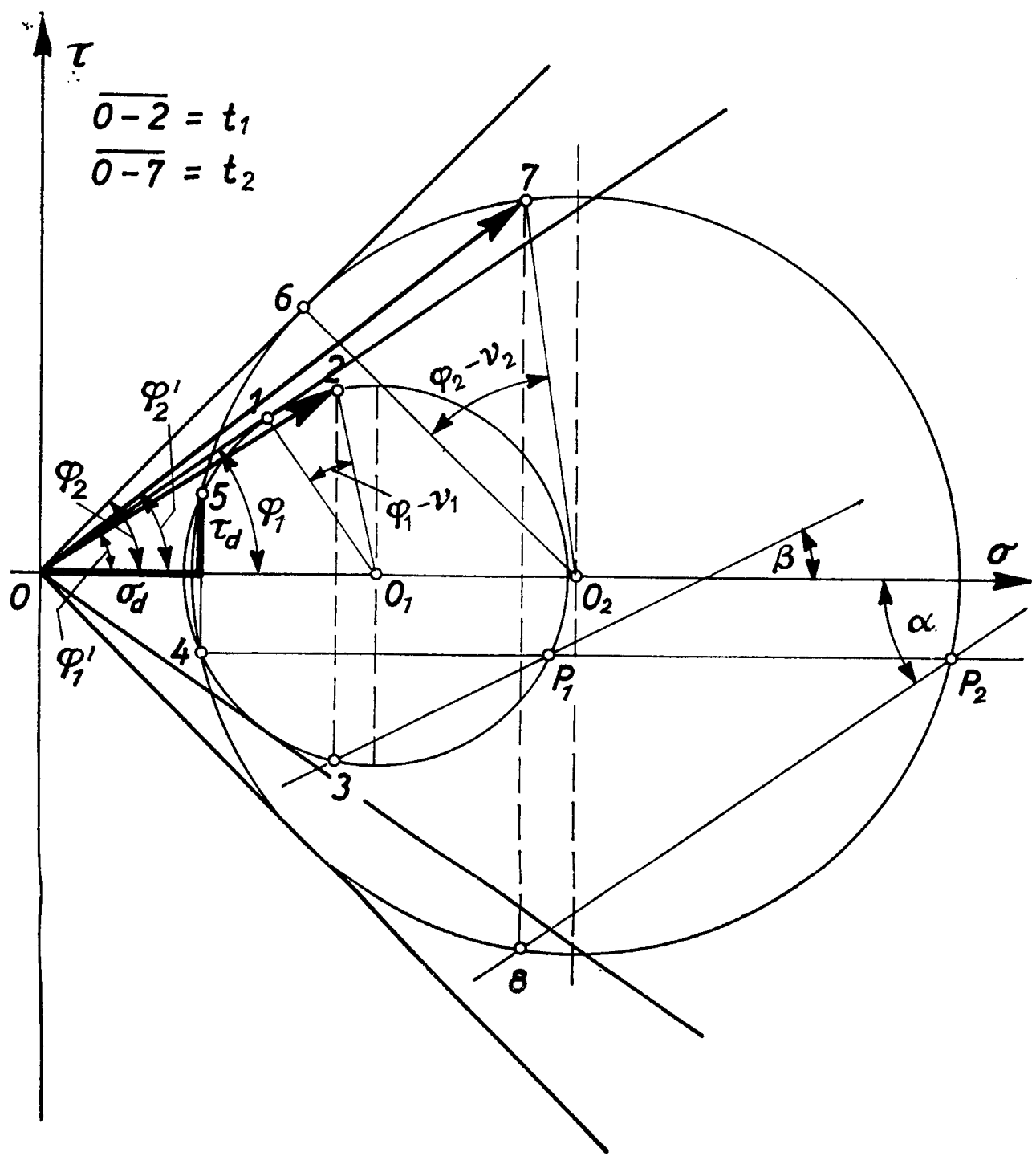

Fig. 4. Graphical solution of the problem

explained in the paper can alternatively by used in order to get the dilatation angles $\nu$ provided that the normal stress in the contact surface be measured as well. The solution can be obtained by trial and error.

\section{NOTATIONS}

$a, b:$ strain characteristics

$m, n:$ stress characteristics

$\phi$ : shear angle

$\phi_{1}$ : inclination of stress vector towards normal to line rupture

$\nu$ : dilatation angle

$\gamma$ : bulk density

$G$ : mass force 
$T: \quad$ surface force

$c, d:$ sizes of the contact surface of two triangular prisms

$\alpha, \beta$ : inclination angles of two triangular prisms

$t:$ reaction stress

$i$ : inclination of the contact surface

$\overrightarrow{p_{d}}$ : stress vector acting on the contact surface of two triangular prisms

$\sigma_{d}:$ normal component of the stress vector $p_{d}$

$\tau_{d}$ : tangential component of the stress vector $p_{d}$

$P$ : pole of the stress circle

\section{REFERENCE}

Hansen, B., (1958), Line Ruptures Regarded as Narrow Rupture Zones, Basic Equations Based on Kinematic Considerations, Brussels Conference 85 on Earth Pressure Problems, Proceedings, Vol. I, pp. 39-48.

(Received June 17, 1969) 